04

\title{
Влияние поляризации считывающей волны и циркулярного дихроизма на дифракционную эффективность отражательной голограммы в кубическом оптически активном фоторефрактивном поглощающем пьезокристалле
}

\author{
(C) В.Н. Навныко ${ }^{1}$, В.В. Шепелевич ${ }^{1}$, С.М. Шандаров ${ }^{2}$ \\ ${ }^{1}$ Мозырский государственный педагогический университет имени И.П. Шамякина, \\ 274760 Мозырь, Республика Беларусь \\ ${ }^{2}$ Томский государственный университет систем управления и радиоэлектроники, \\ 634050 Томск, Россия \\ e-mail: valnav@inbox.ru
}

Поступила в редакцию 25.08.2020 г.

В окончательной редакции 25.08.2020 г.

Принята к публикации 08.09.2020 г.

\begin{abstract}
В приближении медленно меняющихся амплитуд получена система уравнений связанных волн, пригодная для описания дифракции и взаимодействия эллиптически поляризованных световых волн на объемной фазово-амплитудной голографической решетке, сформированной в кубическом оптически активном фоторефрактивном поглощающем пьезокристалле. Изучена зависимость дифракционной эффективности фазовой отражательной голограммы от эллиптичности считывающей волны и толщины кристалла $\mathrm{Bi}_{12} \mathrm{SiO}_{20}$ срезов (001) и (111). Проанализированы закономерности влияния циркулярного дихроизма на дифракционную эффективность фазовой отражательной голограммы. Установлено, что для кристалла среза (001) максимальное значение дифракционной эффективности отражательной голограммы в зависимости от толщины кристалла может достигаться при использовании считывающей волны как с линейной поляризацией, так и с эллиптической поляризацией. Для кристалла среза (111) наибольшая дифракционная эффективность достигается при использовании считывающей волны с линейной поляризацией. Показано, что влияние циркулярного дихроизма на интенсивность восстановленной волны зависит от среза кристалла, эллиптичности считывающей волны и толщины кристалла.
\end{abstract}

Ключевые слова: фоторефрактивный кристалл, отражательная голограмма, дифракционная эффективность, циркулярный дихроизм.

DOI: $10.21883 /$ OS.2021.01.50441.221-20

\section{Введение}

Методы трехмерной оптической голографии впервые предложены Ю.Н. Денисюком. В своих основополагающих работах $[1,2]$ он впервые продемонстрировал возможность восстановления светового пучка с длиной волны, соответствующей длине волны записывающего объемную голограмму излучения. Ю.Н. Денисюком теоретически и экспериментально обоснованы принципы записи объемных отражательных голограмм, проанализированы их свойства и предложена сфера практического применения.

Кубические фоторефрактивные кристаллы $\mathrm{Bi}_{12} \mathrm{SiO}_{20}$ (BSO), $\mathrm{Bi}_{12} \mathrm{TiO}_{20}$ (BTO), $\mathrm{Bi}_{12} \mathrm{GeO}_{20}$ (BGO) являются перспективными средами для записи и считывания объемных голограмм вследствие высокой светочувствительности и относительно малого времени фоторефрактивного отклика [3]. Кристаллы такого типа используются в качестве регистрирующих сред в голографической интерферометрии, оптических корреляторах и фильтрах новизны, а также в устройствах, предназначенных для выполнения оптических логических операций (обзор [4]).
Вклад в модуляцию диэлектрической проницаемости фоторефрактивного кристалла на оптических частотах наряду с линейным электрооптическим эффектом может быть дополнительно внесен [5] за счет совместного действия фотоупругого и обратного пьезоэлектрического эффектов (далее - „пьезоэффект“). Закономерности изменения возмущений тензора диэлектрической непроницаемости сегнетоэлектрического фоторефрактивного кристалла $\mathrm{LiNbO}_{3}$ под действием пьезоэффекта при записи голографической решетки проанализированы в [6]. В работе экспериментально обоснована необходимость учета пьезоэффекта при изучении оптических свойств кристалла. Расчет модуляции диэлектрической проницаемости в кубическом фоторефрактивном кристалле с учетом совместного вклада линейного электрооптического эффекта и пьезоэффекта впервые выполнен в [7]. Аналогичная задача, принимающая во внимание оптическую активность среды, решена в [8]. В работе проанализированы особенности дифракции световой волны на пропускающей фазовой голографической решетке, сформированной в кубическом оптически активном фоторефрактивном кристалле, а также теоре- 
тически и экспериментально изучено влияние пьезоэффекта на зависимость дифракционной эффективности пропускающей голограммы от ориентационного угла. Анализ закономерностей двухволнового взаимодействия на пропускающей голограмме в кубическом оптически активном фоторефрактивном пьезокристалле класса симметрии 23 представлен в работе [9]. Показано, что одновременный учет оптической активности кристалла и пьезоэффекта приводит к согласованию результатов теоретических расчетов и экспериментальных данных. Обзор публикаций по изучению влияния пьезоэффекта на свойства объемных фазовых голограмм в фоторефрактивных кристаллах представлен в [10].

Изучению особенностей дифракции и взаимодействия световых волн на фазовых отражательных голограммах, сформированных в кубических оптически активных фоторефрактивных пьезокристаллах, посвящен ряд исследований (см., например, [11,12]). В [11] представлены полученные с учетом пьезоэффекта соотношения, описывающие в приближении неистощимой накачки двухволновое взаимодействие световых волн в кубических оптически активных фоторефрактивных кристаллах на фазовой отражательной решетке. Показано, что вклад неоднонаправленного энергетического обмена во взаимодействие может обусловливать значительные изменения коэффициента усиления предметной волны. Влияние пьезоэффекта на относительную интенсивность предметной волны и дифракционную эффективность отражательной голограммы, сформированной в кубическом оптически активном фоторефрактивном пьезокристалле, изучено в [12]. Представлены аналитические выражения для нахождения вектора напряженности электрического поля линейно поляризованной предметной волны при встречном двухволновом взаимодействии на фазовой отражательной голограмме. Обзор научных публикаций, посвященных изучению дифракции и взаимодействию световых волн на фазовых отражательных голограммах в кубических оптически активных фоторефрактивных пьезокристаллах, приведен в [13]. В работе представлены результаты, подтверждающие возможность использования объемных фазовых голограмм, записанных в отражательной геометрии, для реализации адаптивных элементов голографических интерферометров.

Как показано в [14], совпадение теоретически рассчитанных графиков зависимости дифракционной эффективности пропускающей голограммы, сформированной в кристалле ВТО среза (1) 10$)$, от ориентационного угла с экспериментальными данными достигается при условии, что голограмма рассматривается как фазовоамплитудная, представляющая собой совокупность фазовой и амплитудной (абсорбционной) голографических решеток. Существование в кристалле ВТО амплитудной голографической решетки обусловлено модуляцией коэффициента поглощения под действием интерференционного светового поля. В [15] теоретически и экспериментально исследована зависимость дифракционной эффективности пропускающей голограммы от толщины кристалла ВТО. Установлено, что наилучшее согласование теоретических и экспериментальных данных достигается при учете фазово-амплитудной структуры пропускающей голограммы, формирующейся в кристалле ВТО. В [16] представлены уравнения связанных волн, предназначенные для расчета компонент векторов напряженностей электрического поля линейно поляризованных световых волн, дифрагирующих на отражательной голограмме в кубическом фоторефрактивном кристалле с естественной оптической активностью, которые получены с учетом вклада амплитудной решетки в двухпучковое взаимодействие. Определена постоянная двухпучковой связи на отражательной голограмме, при которой достигается наилучшее согласование экспериментально измеренной зависимости коэффициента усиления предметной волны от азимутов линейной поляризации световых волн.

Как показывают результаты экспериментальных исследований [17], в нелегированных кубических кристаллах BSO, ВТО и ВGO вследствие наличия вакансий по кремнию, титану и германию соответственно наряду с фотопроводимостью фиксируется наличие заметного циркулярного дихроизма. Вопросы нахождения параметра кругового дихроизма кубического кристалла рассмотрены в [18]. Изучению влияния циркулярного дихроизма на дифракционную эффективность и эффективный коэффициент усиления фазовых пропускающих голограмм, сформированных в кубических оптически активных фоторефрактивных кристаллах, посвящены работы $[19,20]$. Учет циркулярного дихроизма может приводить к существенному изменению дифракционной эффективности пропускающей голограммы, сформированной в кристалле $\mathrm{BSO}$, при ее считывании циркулярно поляризованной волной [19]. Влияние циркулярного дихроизма на эффективный коэффициент усиления пропускающих голограмм, сформированных в кристалле BSO при приложении к нему внешнего постоянного электрического поля, проанализировано в [20]. Показано, что совместное действие поглощения и циркулярного дихроизма в присутствии внешнего электрического поля может обусловливать увеличение эффективного коэффициента усиления и изменение значений ориентационного угла, при которых достигается его максимум.

В настоящее время остается недостаточно изученным вопрос влияния поляризации световых волн на выходные энергетические характеристики (дифракционная эффективность, коэффициент усиления предметной волны) отражательной голограммы, сформированной в кубическом фоторефрактивном поглощающем пьезокристалле, проявляющем естественную оптическую активность и циркулярный дихроизм. Решение этой задачи позволит провести оптимизацию условий считывания голограммы посредством выбора соответствующего сочетания эллиптичности световых волн и толщины кристалла.

Целью настоящей работы является изучение зависимости дифракционной эффективности фазовой отражательной голограммы, сформированной в кубическом 


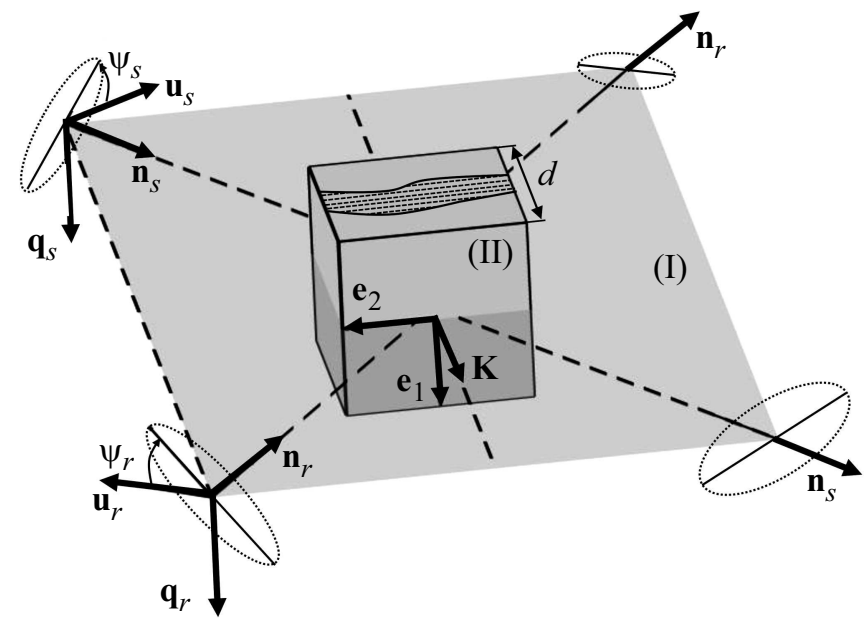

Рис. 1. Схема двухволнового взаимодействия на отражательной голографической решетке, сформированной в кубическом фоторефрактивном кристалле.

оптически активном фоторефрактивном поглощающем пьезокристалле, от эллиптичности считывающей (далее - опорной) волны и толщины кристаллического образца. В работе будут представлены уравнения связанных волн, пригодные для нахождения компонент векторов напряженностей электрических полей эллиптически поляризованных опорной и предметной волн при их взаимодействии на фазово-амплитудной отражательной голограмме. При теоретических расчетах будет приниматься во внимание линейный электрооптический, фотоупругий и обратный пьезоэлектрический эффекты, естественная оптическая активность кристалла, поглощение и циркулярный дихроизм. Будет проанализировано влияние циркулярного дихроизма на зависимость дифракционной эффективности отражательной голограммы от толщины кристалла для различных поляризаций опорной волны и определены условия достижения её максимальных значений.

\section{Теоретическая модель}

Рассмотрим кубический оптически активный фоторефрактивный кристалл класса симметрии 23 толщиной $d$ (рис. 1), в котором сформирована объемная фазовая синусоидальная отражательная голографическая решетка с волновым вектором К. Пусть на кристалл падают две монохроматические плоские эллиптически поляризованные световые волны, опорная $(R)$ и предметная $(S)$. Полагаем, что грани кристалла, на которые падают волны, просветлены, и отраженные волны в кристалле отсутствуют.

Единичные векторы $\mathbf{e}_{1}$ и $\mathbf{e}_{2}$, изображенные на рис. 1, являются ортами рабочей системы координат $\left(\mathbf{e}_{1}, \mathbf{e}_{2}, \mathbf{e}_{3}\right)$, где $\mathbf{e}_{3}=\left[\mathbf{e}_{1} \times \mathbf{e}_{2}\right]$, которая используется для задания ориентации кристаллического образца относительно лабораторного стола. Поверхность лабораторного стола параллельна плоскости (I) и содержит единичные векторы е 2 и $\mathbf{e}_{3}$. Плоскость (II) параллельна плоскости среза кристаллического образца и содержит единичные векторы $\mathbf{e}_{1}$ и $\mathbf{e}_{2}$. Волновой вектор $\mathbf{K}$ перпендикулярен плоскости (II) и направлен в противоположную сторону к вектору е $\mathbf{e}_{3}$. Тройки единичных векторов $\left(\mathbf{q}_{r}, \mathbf{u}_{r}, \mathbf{n}_{r}\right)$ и $\left(\mathbf{q}_{s}, \mathbf{u}_{s}, \mathbf{n}_{s}\right)$ определяют ортонормированные базисы, используемые для задания направлений распространения и описания поляризаций опорной и предметной волн. Единичные векторы $\mathbf{n}_{r}$ и $\mathbf{n}_{s}$ перпендикулярны волновым фронтам и совпадают по направлению с волновыми нормалями опорной и предметной волн соответственно. Пунктирные эллипсы отображают поляризацию волн и перпендикулярны векторам $\mathbf{n}_{r}$ и $\mathbf{n}_{s}$. Сплошные линии внутри эллипсов соответствует их большим полуосям. Азимуты $\psi_{R}$ и $\psi_{S}$ используются для задания ориентации эллипса поляризации опорной и предметной волн. По величине азимуты $\psi_{R}$ и $\psi_{S}$ равны угловым расстояниям между большими полуосями эллипсов и векторами $\mathbf{u}_{r}, \mathbf{u}_{s}$ соответственно и отсчитываются по часовой стрелке, если смотреть в направлении волновой нормали. Параллельными сплошными линиями на рис. 1 показана (в разрезе) объемная голографическая дифракционная решетка, содержащая периодически расположенные рассеивающие волновые фронты плоскости.

Векторы напряженностей электрических полей эллиптически поляризованных опорной и предметной волн в кристалле представим в следующем виде:

$$
\begin{gathered}
\mathbf{R}=\left(R_{1} \mathbf{e}_{r 1}+R_{2} \mathbf{e}_{r 2}\right) \exp \left(i \vartheta_{r}\right), \\
\mathbf{S}=\left(S_{1} \mathbf{e}_{s 1}+S_{2} \mathbf{e}_{s 2}\right) \exp \left(i \vartheta_{s}\right),
\end{gathered}
$$

где $\mathbf{R}$ и $\mathbf{S}$ - комплексные векторные амплитуды опорной и предметной волн; $R_{1,2}$ и $S_{1,2}$ - скалярные составляющие векторных амплитуд $\mathbf{R} \quad$ и $\mathbf{S} ; \quad \mathbf{e}_{r 1, s 1}=\left(\mathbf{q}_{r, s}+i \tau_{r, s} \mathbf{u}_{r, s}\right) / \sqrt{1+\tau_{r, s}^{2}}$, $\mathbf{e}_{r 2, s 2}=\left(\mathbf{u}_{r, s}-i \tau_{r, s} \mathbf{q}_{r, s}\right) / \sqrt{1+\tau_{r, s}^{2}} \quad$ - векторы поляризации; $\mathbf{q}_{r, s}, \mathbf{u}_{r, s}$ - единичные векторы, связанные с геометрией среды, причем $\mathbf{n}_{r, s}=\left[\mathbf{q}_{r, s} \times \mathbf{u}_{r, s}\right] ; \tau_{r, s}-$ параметры эллиптичности опорной и предметной волн; $\vartheta_{r, s}$ - начальные фазы опорной и предметной волн.

При записи голограммы неоднородное световое поле обусловливает фотовозбуждение носителей электрического заряда в фоторефрактивном кристалле, которые в результате диффузии уходят из освещенных областей и захватываются на ловушки в неосвещенных зонах [3]. С использованием одноуровневой зонной модели фоторефрактивного кристалла амплитуда первой гармоники напряженности электрического поля пространственного заряда фоторефрактивного кристалла может быть найдена по следующей формуле [21]:

$$
E_{s c}=-i m \frac{E_{D}}{1+E_{D} / E_{q}},
$$

где $E_{D}=\left(k_{b} T / e\right) K, E_{q}=e N_{A} /(\varepsilon K), K=2 \pi / \Lambda$. Здесь приняты следующие обозначения: $E_{s c}-$ напряженность 
электрического поля пространственного заряда кристалла; $E_{D}$ - напряженность диффузионного электрического поля; $E_{q}$ - напряженность электрического поля насыщения ловушек; $m-$ контраст интерференционной картины; $i$ - мнимая единица; $K$ - модуль волнового вектора голограммы; $k_{b}$ - постоянная Больцмана; $T-$ абсолютная температура; $e-$ элементарный электрический заряд; $N_{A}-$ плотность акцепторов; $\varepsilon-$ диэлектрическая проницаемость кристалла; $\Lambda$ - пространственный период голографической решетки.

При подстановке выражений (1)-(3) в волновое уравнение для оптически активных сред и использовании приближения медленно меняющихся амплитуд можно получить уравнения связанных волн, которые пригодны для описания одновременной дифракции двух эллиптически поляризованных световых волн на фазовоамплитудной голографической решетке, сформированной в кубическом оптически активном фоторефрактивном поглощающем пьезокристалле. В общем виде уравнения связанных волн могут быть представлены как

$$
\begin{gathered}
\frac{d R_{1}}{d z}=\left(i e^{-i \delta} \kappa_{r 1 s 1}+e^{-i \phi} \sigma_{r 1 s 1}\right) S_{1}+\left(i e^{-i \delta} \kappa_{r 1 s 2}\right. \\
\left.+e^{-i \phi} \sigma_{r 1 s 2}\right) S_{2}+\left(\rho_{r} \xi_{r}-\alpha_{r}\right) R_{1}+\left(\rho_{r}+\alpha_{r} \xi_{r}\right) R_{2}, \\
\frac{d R_{2}}{d z}=\left(i e^{-i \delta} \kappa_{r 2 s 1}+e^{-i \phi} \sigma_{r 2 s 1}\right) S_{1}+\left(i e^{-i \delta} \kappa_{r 2 s 2}\right. \\
\left.+e^{-i \phi} \sigma_{r 2 s 2}\right) S_{2}-\left(\rho_{r}+\alpha_{r} \xi_{r}\right) R_{1}+\left(\rho_{r} \xi_{r}-\alpha_{r}\right) R_{2}, \\
\frac{d S_{1}}{d z}=\left(i e^{i \delta} \kappa_{s 1 r 1}+e_{s 1 r 1}^{i \phi}\right) R_{1}+\left(i e^{i \delta} \kappa_{s} 1 r 2+e^{i \phi} \sigma_{s 1 r 2}\right) R_{2} \\
+\left(\rho_{s} \xi_{s}-\alpha_{s}\right) S_{1}+\left(\rho_{s}+\alpha_{s} \xi_{s}\right) S_{2}, \\
\frac{d S_{2}}{d z}=\left(i e^{i \delta} \kappa_{s 2 r 1}+e^{i \phi} \sigma_{s 2 r 1}\right) R_{1}+\left(i e^{i \delta} \kappa_{s 2 r 2}+e^{i \phi} \sigma_{s 2 r 2}\right) R_{2} \\
-\left(\rho_{s}+\alpha_{s} \xi_{s}\right) S_{1}+\left(\rho_{s} \xi_{s}-\alpha_{s}\right) S_{2},
\end{gathered}
$$

где

$$
\begin{gathered}
\kappa_{m k n t}=-\frac{\kappa_{0}\left(\mathbf{e}_{m k}^{*} \Delta \varepsilon^{-1} \mathbf{e}_{n t}\right)}{\cos \varphi_{m}}, \\
\sigma_{m k n t}=-\frac{\sigma_{0}\left(\mathbf{e}_{m k}^{*} \Delta \sigma \mathbf{e}_{n t}\right)}{\cos \varphi_{m}}, \\
\rho_{r, s}=\frac{(\rho+i \chi)}{\cos \varphi_{r, s}}, \quad \xi_{r, s}=\frac{2 i \tau_{r, s}}{\left(1+\tau_{r, s}^{2}\right)}, \alpha_{r, s}=\frac{\alpha}{\cos \varphi_{r, s}} .
\end{gathered}
$$

Приведенные уравнения связанных волн в зависимости от выбора геометрии двухволнового взаимодействия могут использоваться для расчета векторных амплитуд опорной и предметной волн как в случае пропускающей голограммы, так и в случае отражательной голограммы. Геометрия двухволнового взаимодействия и соответственно тип голографической решетки определяются ориентацией ортонормированных базисов $\left(\mathbf{q}_{r}, \mathbf{u}_{r}, \mathbf{n}_{r}\right)$ и $\left(\mathbf{q}_{s}, \mathbf{u}_{s}, \mathbf{n}_{s}\right)$ относительно рабочей системы координат.
В уравнениях связанных волн параметром $\phi$ задан сдвиг фаз между созданной в кристалле интерференционной картиной и фазовой голографической решеткой. В фоторефрактивных кристаллах за счет диффузионного механизма формирования пространственного заряда этот сдвиг фаз может достигать четверти периода $[3,21]$, и в дальнейших расчетах параметр $\phi$ принимается равным $\pi / 2$. Параметр $\sigma$ используется для задания сдвига между интерференционной картиной и амплитудной голографической решеткой, который в общем случае может иметь отличное от нуля значение. Далее в работе будем анализировать случай, когда смещение амплитудной голографической решетки относительно интерференционной картины отсутствует $(\sigma=0)$.

Параметрами $\kappa_{m k n t}$ и $\sigma_{m k n t}$ в уравнениях связанных волн заданы коэффициенты связи между опорной и предметной волнами при их дифракции на фазовой и амплитудной голографических решетках. В выражениях для коэффициентов связи приняты следующие замены: $m=r, s ; n=s, r ; k, t=1,2$. Параметры $\kappa_{0}$ и $\sigma_{0}$ являются постоянными связи фазовой и амплитудной голографических решеток и находятся из следующих выражений: $\kappa_{0}=\pi n^{3} /(2 \lambda), \sigma_{0}=\pi /(n c)$, где $n-$ показатель преломления невозмущенного кристалла, $\lambda$ - длина волны, $c$ - скорость света в вакууме. Тензорные свертки $\left(\mathbf{e}_{m k}^{*} \Delta \varepsilon^{-1} \mathbf{e}_{n t}\right)$ и $\left(\mathbf{e}_{m k}^{*} \Delta \sigma \mathbf{e}_{n t}\right)$ используются для определения вклада в дифракционный процесс взаимной связи между составляющими, на которые распадаются опорная и предметная волны при их распространении в кристалле. В тензорных свертках через $\Delta \varepsilon^{-1}$ и $\Delta \sigma$ обозначены изменения компонент обратного тензора диэлектрической проницаемости и тензора проводимости кристалла. Выражения для нахождения изменения компонент обратного тензора диэлектрической проницаемости кубического кристалла с учетом пьезоэффекта приведены в [22]. Изменение тензора проводимости кубического кристалла в линейном по контрасту $m$ приближении будем рассчитывать на основании следующего выражения: $\Delta \sigma=m \sigma \delta_{k n}$, где $\sigma-$ коэффициент проводимости, $\delta_{k n}$ - единичный симметричный тензор второго ранга. Углы $\varphi_{r}$ и $\varphi_{s}$ (на рис. 1 не показаны) отсчитываются в плоскости I между единичным вектором е 3 и волновыми нормалями $\mathbf{n}_{r}$ и $\mathbf{n}_{s}$. Параметры $\rho, \alpha$ и $\chi$ используются для задания величины удельного вращения, поглощения и циркулярного дихроизма кристалла.

При численных расчетах рассматривался кристалл с параметрами $\mathrm{BSO}$, заимствованными из работ [3,23-25]: показатель преломления невозмущенного кристалла $n_{0}=2.54$ при $\lambda=633 \cdot 10^{-9} \mathrm{~m}[3]$; электрооптический коэффициент $r_{41}=-5 \cdot 10^{-12} \mathrm{~m} / \mathrm{V}$ [3]; коэффициенты упругости $c_{1}=12.96 \cdot 10^{10} \mathrm{~N} / \mathrm{m}^{2}, c_{2}=2.99 \cdot 10^{10} \mathrm{~N} / \mathrm{m}^{2}$, $c_{3}=2.45 \cdot 10^{10} \mathrm{~N} / \mathrm{m}^{2}[23]$; коэффициенты фотоупругости $p_{1}=-0.16, p_{2}=-0.13, p_{3}=-0.12, p_{4}=-0.015[24]$; пьезоэлектрический коэффициент $e_{14}=1.12 \mathrm{C} / \mathrm{m}^{2}[23]$. Здесь приняты следующие обозначения для ненулевых компонент тензоров линейного электрооптического $\left(\hat{r}^{S}\right)$, фотоупругого $\left(\hat{p}^{E}\right)$ и обратного пьезоэлектрического $(\hat{e})$ 
эффектов, а также компонент тензора упругости $\left(\hat{c}^{E}\right)$ : $r_{123}^{S}=r_{132}^{S}=r_{213}^{S}=r_{231}^{S}=r_{312}^{S}=r_{321}^{S} \equiv r_{41}, p_{11}^{E}=p_{22}^{E}=$ $=p_{33}^{E} \equiv p_{1}, p_{12}^{E}=p_{23}^{E}=p_{31}^{E} \equiv p_{2}, p_{13}^{E}=p_{21}^{E}=p_{32}^{E} \equiv p_{3}$, $p_{44}^{E}=p_{55}^{E}=p_{66}^{E} \equiv p_{4}, \quad e_{123}=e_{132}=e_{213}=e_{231}=e_{312}=$ $=e_{321} \equiv e_{14}, \quad c_{11}^{E}=c_{22}^{E}=c_{33}^{E} \equiv c_{1}, c_{12}^{E}=c_{13}^{E}=c_{23}^{E}=$ $=c_{21}^{E}=c_{31}^{E}=c_{32}^{E} \equiv c_{2}, c_{44}^{E}=c_{55}^{E}=c_{66}^{E} \equiv c_{3}$. Индекс $S$ для тензора линейного электрооптического эффекта $\hat{r}^{S}$ означает, что компонента $r_{41}$ тензора линейного электрооптического эффекта измерялась для зажатого кристалла; компоненты тензоров упругости $\hat{c}^{E}$ и фотоупругого эффекта $\hat{p}^{E}$ измерялись при постоянном электрическом поле. При расчетах использовались следующие численные значения физических величин: $\rho=372 \mathrm{rad} / \mathrm{m}[3,9], \chi=10 \mathrm{~m}^{-1}[20], \alpha=53 \mathrm{~m}^{-1}$ [25], $N_{A}=10^{22} \mathrm{~m}^{-3}$ [26], $\varepsilon=56$ [26], $T=300 \mathrm{~K}, \varphi_{r}=-5^{\circ}$, $\varphi_{s}=185^{\circ}$. Отношение интенсивностей опорной и предметной волн принималось равным $1: 20$.

Для характеристики степени восстановления предметной волны при дифракции опорной волны на отражательной голограмме используется дифракционная эффективность голограммы, которая находится на основании следующего выражения: $\eta=\left(I_{d} / I_{r}\right) 100 \%$, где $I_{d}$ - интенсивность предметной волны на выходе из кристалла, $I_{r}-$ интенсивность опорной волны на входе в кристалл.

\section{Результаты и их анализ}

На рис. 2, a изображен график зависимости дифракционной эффективности $\eta$ отражательной голограммы от толщины $d$ кристалла BSO среза (001) и эллиптичности $\tau_{r}$ опорной волны. Максимум дифракционной эффективности кристалла в зависимости от эллиптичности опорной волны достигается в интервале $2.6<d<4.2 \mathrm{~mm}$. Наибольшая дифракционная эффективность $(\eta=2.9 \%)$ имеет место при линейной поляризации опорной волны в кристалле толщиной $4.1 \mathrm{~mm}$ (точка $A_{1}$ ). С увеличением эллиптичности интенсивность восстановленной предметной волны в максимуме уменьшается и достигается в более тонких кристаллических образцах. Для циркулярно поляризованной опорной волны максимум $(\eta=1.2 \%)$ достигается в кристалле толщиной $2.6 \mathrm{~mm}$ (точка $B_{1}$ ). Наименьшая дифракционная эффективность $(\eta=0.2 \%)$ на поверхности $\eta\left(d, \tau_{r}\right)$ достигается при толщине кристалла $5.6 \mathrm{~mm}$ для опорной волны с циркулярной поляризацией (точка $B_{2}$ ).

Изменение эллиптичности обусловливает качественную трансформацию графика зависимости, представленного на рис. 2, $a$. Если эллиптичность меньше 0.5 , поверхность $\eta\left(d, \tau_{r}\right)$ имеет бугристый вид: максимумы дифракционной эффективности (точки $A_{1}, A_{2}, A_{3}$ и $A_{4}$ ) достигаются при различных значениях толщины кристалла. Для остальных значений эллиптичности максимумы достигаются при приблизительно одинаковых
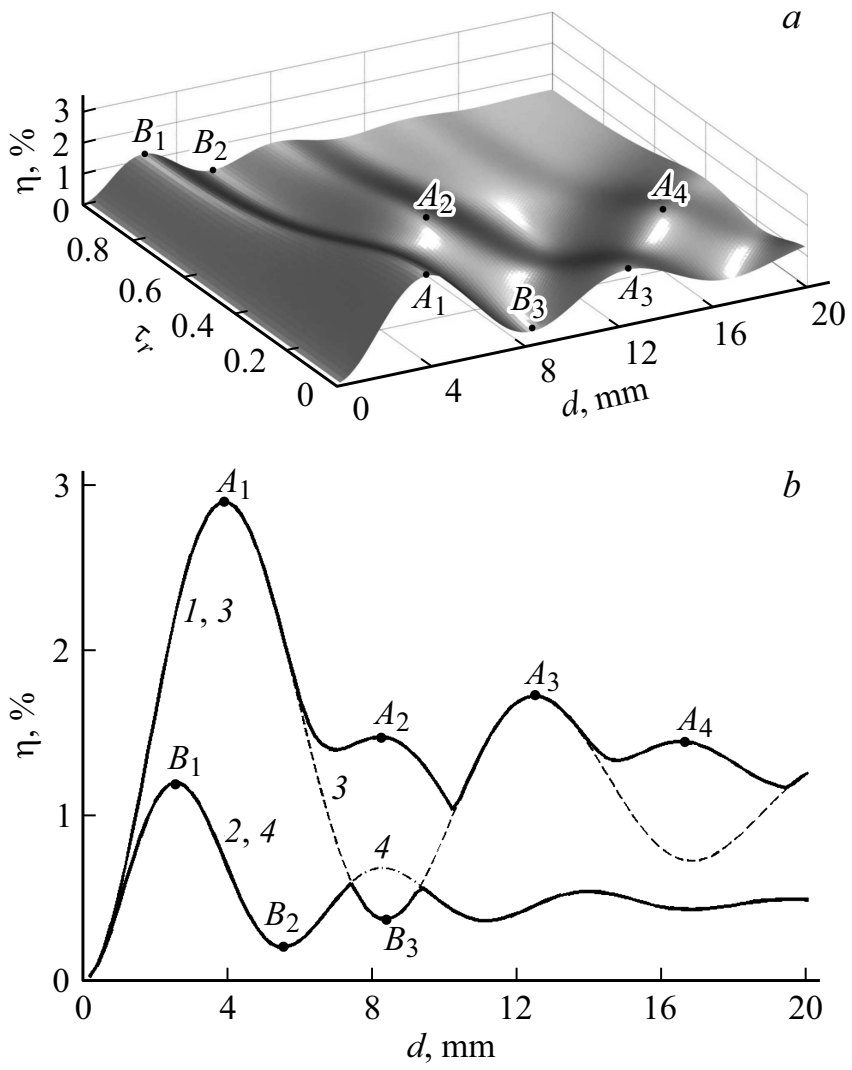

Рис. 2. $a-$ зависимость дифракционной эффективности $\eta$ отражательной голограммы, сформированной в кристалле BSO среза (001), от толщины $d$ и эллиптичности $\tau_{r} ; b-$ зависимости максимальных (кривая 1) и минимальных (кривая 2) значений дифракционной эффективности отражательной голограммы от толщины и зависимости $\eta(d)$, рассчитанные при $\tau_{r}=0$ (кривая 3) и $\tau_{r}=1$ (кривая 4).

значениях толщины кристалла, в результате чего поверхность $\eta\left(d, \tau_{r}\right)$ приобретает волнообразный вид.

На рис. $2, b$ сплошные линии соответствуют графикам зависимости максимальных (кривая 1) и минимальных (кривая 2) значений дифракционной эффективности отражательной голограммы от толщины кристалла BSO среза (001). Построение графиков осуществлялось по следующей схеме: при фиксированной толщине кристалла рассчитывались значения дифракционной эффективности с шагом по эллиптичности 0.01. Далее фиксировались максимальные и минимальные значения, и процедура повторялась для остальных значений толщины кристалла. В результате для каждой толщины кристалла находятся максимальные и минимальные значения дифракционной эффективности, которые и отображаются в виде графиков. На рисунке также дополнительно представлены графики зависимости дифракционной эффективности от толщины кристалла, рассчитанные для опорной волны с линейной (штриховая линия, кривая 3) и циркулярной (штрихпунктирная линия, кривая 4) поляризациями. 
При толщине кристалла до $6 \mathrm{~mm}$ максимальная дифракционная эффективность достигается при использовании опорной волны с линейной поляризацией. Её наибольшее значение в указанном интервале достигается при толщине кристалла $4.1 \mathrm{~mm}$. В этом случае поворот плоскости линейной поляризации опорной волны при ее распространении в кристалле стремится к $90^{\circ}$ $(d \approx \pi /(2 \rho))$ и имеет место когерентное суммирование парциальных волн, возникших при дифракции опорной волны на отражательной голограмме.

В интервале $6<d \leq 10.4 \mathrm{~mm}$ становится выгодным использование эллиптически поляризованной опорной волны. Наибольшее значение дифракционной эффективности достигается для кристалла толщиной $8.2 \mathrm{~mm}$ при эллиптичности опорной волны 0.42 (точки $A_{2}$ ). Использование опорной волны с линейной поляризацией при толщине кристалла $8.2 \mathrm{~mm}$ приводит к достижению минимально возможной интенсивности восстановленной предметной волны (точка $B_{3}$ ), поскольку такая толщина соответствует углу поворота плоскости линейной поляризации опорной волны $180^{\circ}(d \approx \pi / \rho)$ и возникшие при дифракции опорной волны на голограмме парциальные волны когерентно вычитаются.

Если толщина кристалла более $10.4 \mathrm{~mm}$, наибольшие значения дифракционной эффективности достигаются в кристалле толщиной $12.8 \mathrm{~mm}$ (точка $A_{3}$ ) при использовании опорной волны с линейной поляризацией, а также в кристалле толщиной $16.4 \mathrm{~mm}$ (точка $A_{4}$ ) при использовании опорной волны с эллиптичностью 0.24. Выбор поляризации для достижения наибольшей дифракционной эффективности зависит от толщины кристалла: в интервалах $10.4<d \leq 14 \mathrm{~mm}$ и $19.6<d \leq 20 \mathrm{~mm}$ выгодно использовать опорную волну с линейной поляризацией, а в интервале $14<d \leq 19.6 \mathrm{~mm}$ - с эллиптической поляризацией.

Наименьшие значения дифракционной эффективности для всех значений толщины кристалла, за исключением интервала $7.4<d \leq 9.4 \mathrm{~mm}$, достигаются при использовании опорной волны с циркулярной поляризацией. В интервале $7.4<d \leq 9.4 \mathrm{~mm}$ наименьшая дифракционная эффективность достигается при использовании опорной волны с линейной поляризацией.

Для изучения влияния циркулярного дихроизма на интенсивность восстановленной предметной волны введем параметр $\Delta$, который находится путем вычитания значений дифракционной эффективности, рассчитанных с учетом и без учета циркулярного дихроизма. На рис. 3 в прямоугольной системе координат представлены тоновые графики зависимости $\Delta\left(d, \tau_{r}\right)$, которые иллюстрируют области положительных и отрицательных значений параметра $\Delta$ в зависимости от толщины кристалла и эллиптичности опорной волны. Если дифракционная эффективность, рассчитанная с учетом циркулярного дихроизма, равна или превышает значение дифракционной эффективности $(\Delta \geq 0)$, рассчитанное без учета циркулярного дихроизма, то точка на тоновом
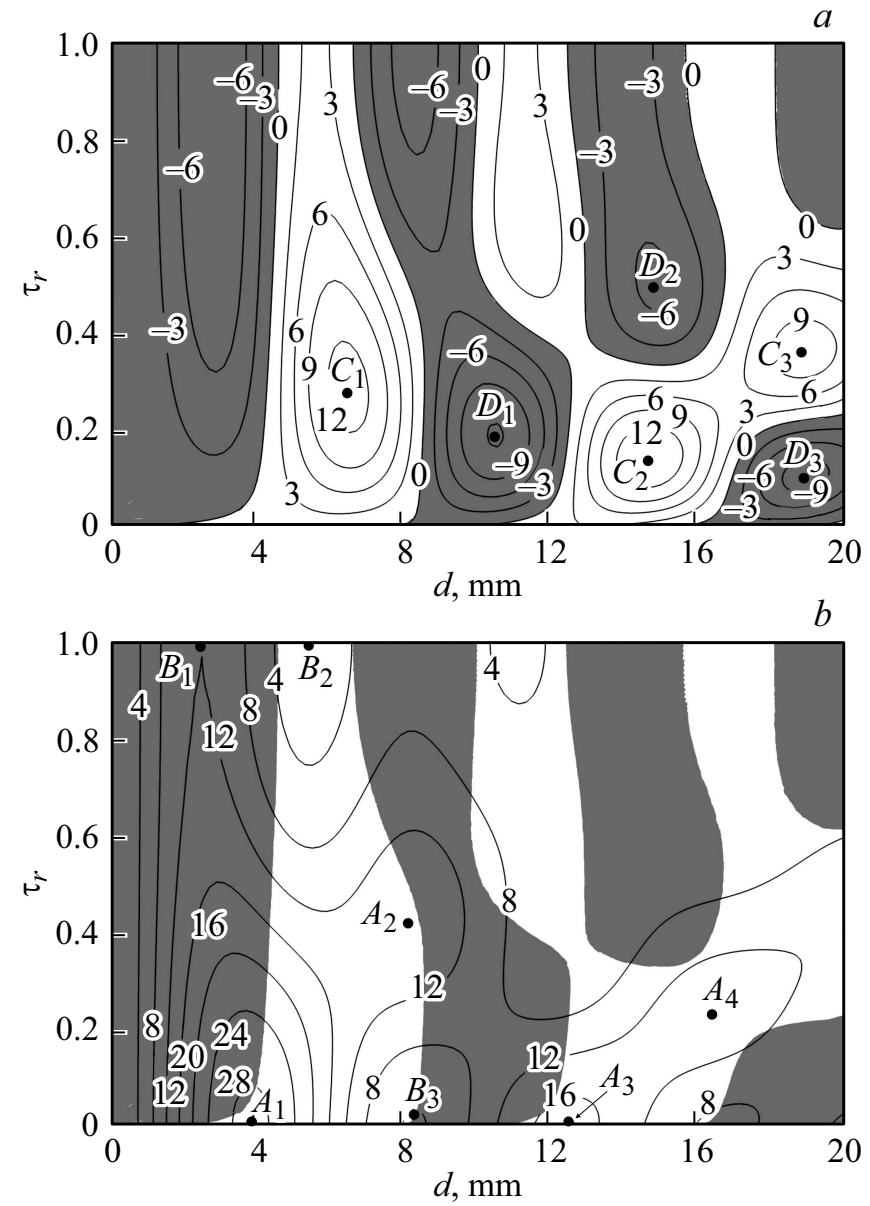

Рис. 3. Зависимость разности $\Delta$ между значениями дифракционной эффективности отражательной голограммы, рассчитанными для кристалла BSO среза (001) с учетом и без учета циркулярного дихроизма, от толщины $d$ и эллиптичности $\tau_{r}$. На рис. $3, a$ контурные линии соединяют точки с одинаковыми значениями $\Delta \cdot 10^{2}$; на рис. $3, b$ контурные линии соединяют точки с одинаковыми значениями $\eta \cdot 10$ графика зависимости $\eta\left(d, \tau_{r}\right)$, представленного на рис. $2, a$.

рисунке окрашивается в белый цвет, в противном случае $(\Delta<0)$ - в серый цвет. Как видно из построения, интервалы значений толщины кристалла, при которых циркулярный дихроизм увеличивает дифракционную эффективность, являются наибольшими при использовании линейно поляризованной волны. При увеличении эллиптичности опорной волны такие интервалы значений толщины кристалла существенно уменьшаются.

На рис. 3, $a$ контурными линиями дополнительно отмечены точки на тоновом рисунке с одинаковыми значениями $\Delta$. Приведенные рядом с контурными линиями цифры соответствуют умноженному на $10^{2}$ параметру $\Delta$. Как видно из построения, максимальные изменения дифракционной эффективности при „включении“ циркулярного дихроизма имеет место при $\tau_{r} \leq 0.5$. Наибольшее увеличение дифракционной эффективности при учете циркулярного дихроизма достигается в 
точках $C_{1}\left(d=6.6 \mathrm{~mm}, \tau_{r}=0.28\right), C_{2}(d=14.6 \mathrm{~mm}$, $\left.\tau_{r}=0.14\right)$ и $C_{3}\left(d=18.8 \mathrm{~mm}, \tau_{r}=0.36\right)$, а наибольшее уменьшение - в точках $D_{1},\left(d=10.6 \mathrm{~mm}, \tau_{r}=0.19\right)$, $D_{2}\left(d=15 \mathrm{~mm}, \tau_{r}=0.5\right), D_{3} \quad\left(d=19 \mathrm{~mm}, \tau_{r}=0.11\right)$. При некоторых значениях толщины кристалла вклад циркулярного дихроизма в дифракционные процессы можно регулировать за счет изменения эллиптичности. Например, для кристалла толщиной $15 \mathrm{~mm}$ увеличение эллиптичности приводит к росту параметра $\Delta$, который достигает максимума при $\tau_{r}=0.14$. С дальнейшим увеличением эллиптичности параметр $\Delta$ начинает уменьшаться. При $\tau_{r}>0.3$ параметр $\Delta$ переходит в область отрицательных значений с минимумом, достигаемым при $\tau_{r}=0.5$.

На рис. $3, b$ посредством контурных линий дополнительно изображен график зависимости $\eta\left(d, \tau_{r}\right)$, приведенный на рис. 2, $a$. Контурные линии объединяют точки с одинаковыми значениями дифракционной эффективности, которые приведены рядом с контурными линиями и умножены на 10. Циркулярный дихроизм обусловливает увеличение дифракционной эффективности в точках максимумов поверхности $\eta\left(d, \tau_{r}\right)$ (точки $A_{1}, A_{2}, A_{3}, A_{4}$ ), a также в точке $B_{2}$. Под действием циркулярного дихроизма имеет место уменьшение дифракционной эффективности в точке минимума $B_{1}$. Точка $B_{2}$ лежит на границе между светлой и серой областями, что означает минимальное влияние циркулярного дихроизма на дифракционную эффективность.

В экспериментах по считыванию отражательной голограммы в кубическом фоторефрактивном кристалле наряду с кристаллическими образцами среза $\{100\}$ также используются образцы среза $\{111\}$ [11-13]. Это обусловлено тем, что для кристаллических образцов среза $\{100\}$ с увеличением толщины кристалла оптическая активность приводит к уменьшению дифракционной эффективности отражательной голограммы и относительной интенсивности предметной волны при встречном двухволновом взаимодействии. Для кристаллов среза $\{111\}$ оптическая активность влияет на выходные энергетические характеристики отражательной голограммы в меньшей степени, и их значения растут с увеличением толщины кристалла.

На рис. 4, a изображен график зависимости дифракционной эффективности $\eta$ отражательной голограммы от толщины $d$ кристалла BSO среза (111) и эллиптичности $\tau_{r}$ опорной волны. Поверхность графика зависимости $\eta\left(d, \tau_{r}\right)$, рассчитанная для кристалла среза (111), имеет более гладкий вид, чем аналогичная поверхность (рис. 2,a), рассчитанная для кристалла среза (100). Максимальное значение дифракционной эффективности (точка $A_{1}$ ) достигается для линейной поляризации опорной волны $\left(\tau_{r}=0\right)$ при наибольшей толщине кристалла $(d=20.0 \mathrm{~mm})$. Точки $A_{2}, A_{3}$ и $A_{4}$ соответствуют максимумам дифракционной эффективности, достигаемым при использовании опорной волны с эллиптичностями $0.25,0.5$ и 1 соответственно.
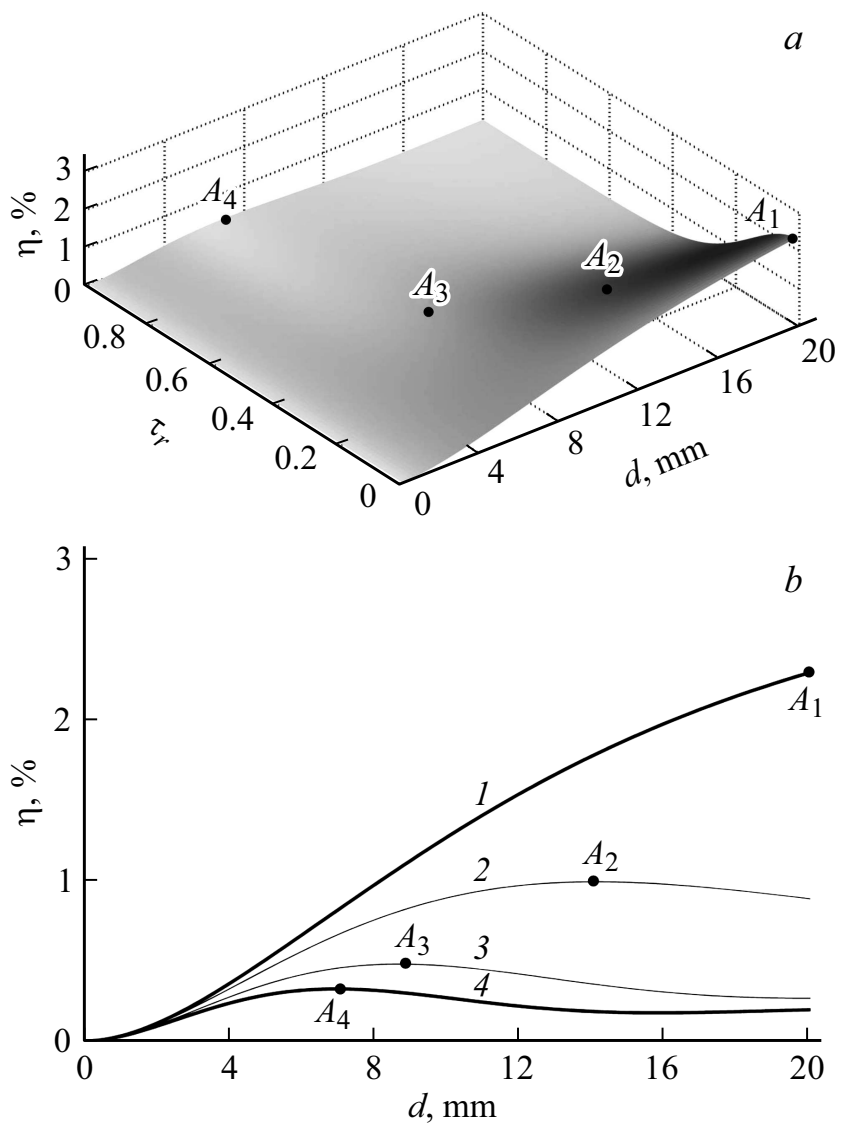

Pис. 4. $a-$ зависимость дифракционной эффективности $\eta$ отражательной голограммы, сформированной в кристалле BSO среза (111), от толщины $d$ и эллиптичности $\tau_{r} ; b$ - зависимости дифракционной эффективности $\eta$ отражательной голограммы от толщины кристалла $d$, рассчитанные при значениях эллиптичности опорной волны $\tau_{r}=0(1), 0.25$ (2), 0.5 (3), 1 (4).

На рис. 4, $b$ сплошные линии соответствуют максимальным (кривая 1) и минимальным (кривая 4) значениям дифракционной эффективности отражательной голограммы, сформированной в кристалле BSO среза (111). Кривые 2 и 3 на рис. 4, $b$ отображают графики зависимости дифракционной эффективности отражательной голограммы от толщины кристалла, рассчитанные при $\tau_{r}=0.25$ и $\tau_{r}=0.5$ соответственно. Видно, что при любой толщине кристалла в интервале $0<d \leq 20 \mathrm{~mm}$ наибольшая дифракционная эффективность достигается при использовании опорной волны с линейной поляризацией, а наименьшая - с циркулярной поляризацией. С увеличением эллиптичности наибольшая дифракционная эффективность достигается в более тонких кристаллах: $A_{1}(20 \mathrm{~mm}) \rightarrow A_{2}(14.3 \mathrm{~mm}) \rightarrow A_{3}(8.6 \mathrm{~mm}) \rightarrow A_{4}(7 \mathrm{~mm})$.

Как следует из сравнения рис. $2, b$ и рис. $4, b$, при толщине кристалла до $9.6 \mathrm{~mm}$ наибольшая дифракционная эффективность достигается при использовании кристалла среза (100). При этом для кристалла толщиной до $7 \mathrm{~mm}$ следует использовать опорную волну с линейной поляризацией, а в интервале от 7 до $9.6 \mathrm{~mm}-$ 

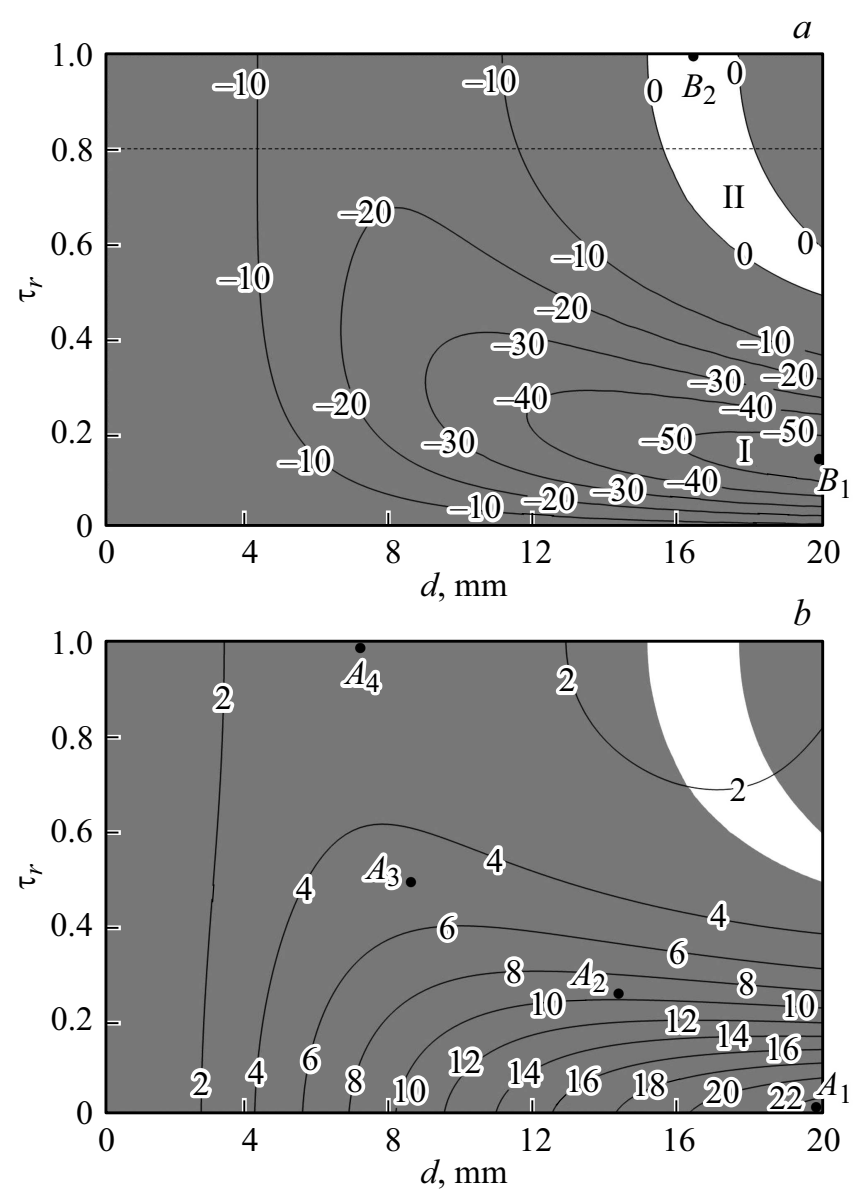

Рис. 5. Зависимость разности $\Delta$ между значениями дифракционной эффективности отражательной голограммы, рассчитанными для кристалла BSO среза (111) с учетом и без учета циркулярного дихроизма, от толщины $d$ и эллиптичности $\tau_{r}$. $a$ - контурные линии соединяют точки с одинаковыми значениями $\Delta \cdot 10^{2} ; b-$ контурные линии соединяют точки с одинаковыми значениями $\eta \cdot 10$ графика зависимости $\eta\left(d, \tau_{r}\right)$, представленного на рис. $4, a$.

волну с эллиптической поляризацией. В интервале от 9.6 до $20 \mathrm{~mm}$ наибольшая дифракционная эффективность достигается при считывании отражательной голограммы опорной волной с линейной поляризацией. Если толщина в этом интервале изменяется от 11 до $13 \mathrm{~mm}$, то наибольшая дифракционная эффективность достигается в кристалле среза (100), а для остальных значений толщины - в кристалле среза (111). Наименьшие значения дифракционной эффективности при толщине кристалла до $4.6 \mathrm{~mm}$ достигаются в кристалле среза (111) при использовании опорной волны с линейной поляризацией, а для остальных значений толщины - в кристалле среза (100).

На рис. 5, а представлен рассчитанный для кристалла BSO среза (111) тоновый график зависимости $\Delta\left(d, \tau_{r}\right)$ c нанесенными на него контурными линиями, которые объединяют точки с одинаковыми значениями $\Delta \times 10^{2}$. При эллиптичности менее 0.5 учет циркулярного дихро- изма обусловливает уменьшение дифракционной эффективности для кристалла любой толщины. Наибольшее уменьшение достигается в области I и в минимуме равняется $\Delta=-5.5 \cdot 10^{-2}$ (точка $\left.B_{1}\right)$. При $\tau_{r}>0.5$ существуют такие значения эллиптичности опорной волны и толщины кристалла (область II), при которых имеет место увеличение дифракционной эффективности под действием циркулярного дихроизма. Наибольшее увеличение дифракционной эффективности достигается в точке $B_{2}$ и составляет $\Delta=6.35 \cdot 10^{-2}$.

На рис. $5, b$ нанесены контурные линии, объединяющие точки с одинаковыми значениями $\eta \times 10$ графика зависимости $\eta\left(d, \tau_{r}\right)$, представленного на рис. 4, $a$. Видно, что при учете циркулярного дихроизма уменьшается дифракционная эффективность в максимумах графиков $\eta(d)$. Как следует из сравнения рис. 5, $a$ и рис. $5, b$, при учете циркулярного дихроизма изменение дифракционной эффективности в максимумах графиков зависимостей $\eta(d)$, рассчитанных для различных поляризаций опорной волны, уменьшается с увеличением эллиптичности опорной волны.

\section{Заключение}

В приближении медленно меняющихся амплитуд получена система уравнений связанных волн, которую можно использовать для описания дифракции и взаимодействия эллиптически поляризованных световых волн на объемной фазово-амплитудной голографической решетке, сформированной в кубическом оптически активном фоторефрактивном кристалле класса симметрии 23. При выводе уравнений учитывались линейный электрооптический, фотоупругий, обратный пьезоэлектрический эффекты, оптическая активность, естественное поглощение кристалла и циркулярный дихроизм.

Теоретически получены и проанализированы зависимости дифракционной эффективности фазовых отражательных голограмм Денисюка, сформированных в кристалле $\mathrm{Bi}_{12} \mathrm{SiO}_{20}$ срезов (001) и (111), от толщины кристаллического образца и эллиптичности опорной волны. Изучено влияние циркулярного дихроизма на дифракционную эффективность фазовой отражательной голограммы. Определены оптимальные условия считывания отражательных голограмм, сформированных в кристалле $\mathrm{Bi}_{12} \mathrm{SiO}_{20}$ срезов (001) и (111), для достижения наибольшей интенсивности восстановленной предметной волны.

Наибольшая дифракционная эффективность фазовой отражательной голограммы достигается в кристалле $\mathrm{Bi}_{12} \mathrm{SiO}_{20}$ среза (001) толщиной $4.1 \mathrm{~mm}$ при использовании опорной волны с линейной поляризацией. При толщине кристалла до $9.6 \mathrm{~mm}$ наибольшая дифракционная эффективность достигается при использовании кристалла среза (001). В интервале от 9.6 до $20 \mathrm{~mm}$ наибольшая дифракционная эффективность достигается при считывании отражательной голограммы опорной 
волной с линейной поляризацией. Если толщина в этом интервале изменяется от 11 до $13 \mathrm{~mm}$, то наибольшая дифракционная эффективность достигается в кристалле среза (001), а для остальных значений толщины - в кристалле среза (111).

Для кристалла $\mathrm{Bi}_{12} \mathrm{SiO}_{20}$ среза (001) наибольшие изменения дифракционной эффективности при „включении“ циркулярного дихроизма достигаются, если эллиптичность опорной волны менее 0.5. Для кристалла $\mathrm{Bi}_{12} \mathrm{SiO}_{20}$ среза (111) учет циркулярного дихроизма обусловливает уменьшение дифракционной эффективности в максимумах графиков зависимостей $\eta(d)$, рассчитанных для любых значений эллиптичности опорной волны.

\section{Финансирование работы}

Работа выполнена при поддержке Министерства образования Республики Беларусь в рамках задания 1.2.01 Государственной программы научных исследований „Фотоника, опто- и микроэлектроника“ на 2016-2020 гг. и Министерства науки и высшего образования Российской Федерации в рамках Госзадания на 2020-2023 гг. (задание FEWM-2020-0038/3).

\section{Конфликт интересов}

Авторы заявляют, что у них нет конфликта интересов.

\section{Список литературы}

[1] Денисюк Ю.Н. // Докл. АН СССР. 1962. Т. 144. № 6. C. 1275.

[2] Денисюк Ю.Н. // Опт. и спектр. 1963. Т. 15. № 4. С. 522.

[3] Петров М.П., Степанов С.И., Хоменко. А.В. Фоторефрактивные кристаллы в когерентной оптике. СПб.: Наука, 1992. $320 \mathrm{c}$.

[4] Stepanov S.I. // Rep. Prog. Phys. 1994. V. 57. N 1. P. 39.

[5] Изванов А.А., Мандель А.Е., Хатьков Н.Д., Шандаров С.М. // Автометрия. 1986. Т. 2. С. 79.

[6] Mandel A.E., Khatkov N.D., Shandarov S.M. // Ferroelectrics. 1988. V. 83. P. 215.

[7] Степанов С.И., Шандаров С.М., Хатьков Н.Д. // ФТТ. 1987. T. 10. C. 3054.

[8] Shepelevich V.V., Shandarov S.M., Mandel A.E. // Ferroelectrics. 1990. V. 110. P. 235.

[9] Shepelevich V.V., Egorov N.N., Shepelevich Victor V. // J. Opt. Soc. Am. B. 1994. V. 11. N 8. P. 1394.

[10] Шепелевич В.В. // Журн. прикл. спектр. 2011. Т. 78. № 4. C. 493; Shepelevich V.V. // J. Appl. Spectrosc. 2011. V. 78. N 4. P. 461.

[11] Мартьянов А.Г., Шандаров С.М., Литвинов Р.В. // ФТТ. 2002. T. 44. № 6. C. 1006; Mart'yanov A.G., Shandarov S.M., Litvinov R.V. // Phys. of the Solid State. 2002. V. 44. N 6. P. 1050.

[12] Шепелевич В.В., Навныко В.Н., Ничипорко С.Ф., Шандаров С.М., Мандель А.Е. // Письма в ЖТФ. 2003. Т. 29. B. 18. C. 22; Shepelevich V.V., Navnyko V.N., Nichiporko S.F., Shandarov S.M., Mandel' A.E. // Tech. Phys. Lett. 2003. V. 29. N 9. P. 757.
[13] Шандаров С.М., Буримов Н.И., Кульчин Ю.Н., Ромашко Р.В., Толстик А.Л., Шепелевич В.В. // Квант. электрон. 2008. Т. 38. № 11. C. 1059; Shandarov S.M., Burimov N.I., Kul'chin Yu.N., Romashko R.V., Tolstik A.L., Shepelevich V.V. // Quant. Electron. 2008. V. 38. N 11. P. 1059.

[14] Шепелевич В.В., Макаревич А.В., Шандаров С.М. // Письма в ЖТФ. 2014. Т. 40. В. 22. С. 83; Shepelevich V.V., Makarevich A.V., Shandarov S.M. // Tech. Phys. Lett. 2014. V. 40. N 11. P. 1024.

[15] Макаревич А.В., Шепелевич В.В., Шандаров С.М. // ЖТФ. 2017. T. 87. B. 5. C. 766; Makarevich A.V., Shepelevich V.V., Shandarov S.M. // Tech. Phys. 2017. V. 62. N 5. P. 785.

[16] Плесовских А.М., Шандаров С.М., Мартьянов А.Г., Мандель А.Е., Буримов Н.И., Шаганова Е.А., Каргин Ю.Ф., Волков В.В., Егорышева А.В. // Квант. электрон. 2005. T. 35. № 2. C. 163; Plesovskikh A.M., Shandarov S.M., Mart'yanov A.G., Mandel' A.E., Burimov N.I., Shaganova E.A., Kargin Yu.F., Volkov V.V., Egorysheva A.V. // Quant. Electron. 2005. V. 35. N 2. P. 163.

[17] Бурков В.И., Каргин Ю.Ф., Кизель В.А., Ситникова В.И., Скориков В.М. // Письма в ЖЭТФ. 1988. Т. 38. № 7. C. 326; Burkov V.I., Kargin Yu.F., Kizel' V.A., Sitnikova V.I., Skorikov V.M. // JEPT Lett. 1983. V. 38. N 7. P. 390.

[18] Бокуть Б.В., Митюрич Г.С., Шепелевич В.В. // Докл. АН БССР. 1979. Т. 23. № 6. С. 507.

[19] Khomutovski P.P., Shepelevich V.V. // Proc. SPIE. 1998. V. 3347. P. 84.

[20] Фирсов А.А., Шепелевич В.В. // Веснік Мазырскага дзяржаўнага педагагічнага ўніверсітэта. 2005. № 1. С. 21.

[21] Kukhtarev N.V., Markov V.B., Odulov S.G., Soskin M.S., Vinetskii V.L. // Ferroelectrics. 1979. V. 22. P. 949.

[22] Шандаров С.М., Шепелевич В.В., Хатьков Н.Д. // Опт. и спектр. 1991. Т. 70. № 5. С. 1068; Shandarov S.M., Shepelevich V.V., Khatkov N.D. // Opt. Spectrosc. 1991. V. 70. N 5. P. 627.

[23] Александров К.С., Бондаренко В.С., Зайщева М.П., Сорокин Б.П., Кокорин Ю.И., Зражсевский В.М., Соболев Б.В. // ФТТ. 1984. Т. 26. № 12. С. 3603.

[24] Бабонас Г.А., Реза А.А., Леонов Е.И., Шандарис В.И. // ЖТФ. 1985. Т. 55. № 6. С. 1203.

[25] Woods C.L., Matson C.L., Salour M.M. // Appl. Phys. A. 1986. V. A40. P. 177.

[26] Solymar L., Webb D.J., Grunnet-Jepsen A. The Physics and Applications of Photorefractive Materials. Oxford: Clarendon press, 1996. $493 \mathrm{p}$. 